This paper is a postprint of a paper submitted to and accepted for publication in Electronics Letters and is subject to Institution of Engineering and Technology Copyright. The copy of record is available at IET Digital Library 


\section{Rapid wide-field heterodyne interferometry with custom 2D CMOS camera}

\author{
J. Zhang, C.W. See, R.J. Smith, N.S. Johnston, M.C. Pitter, \\ M.G. Somekh and R.A. Light
}

A wide-field pseudo-heterodyne interference contrast microscope is described, which employs a complementary metal-oxide semiconductor (CMOS) phase-sensitive camera. The use of multiple wells in the camera enables extremely rapid measurement of a full phase field at high resolution and the modulation frequency is not limited by the camera frame rate. The high data acquisition frequency allows the effects of microphonics to be frozen to mitigate the effect of lowfrequency disturbance.

Introduction: Phase imaging techniques, such as phase contrast and differential interference contrast microscopy [1, 2], are powerful tools to image low contrast phase objects without sample perturbation. These methods generally produce high contrast images; however, they are not quantitative unless special measures are taken. In addition, quantitative interference microscopes generally use non-common path setups, which make their widespread application problematic as overcoming microphonic vibrations leads to bulky and expensive implementations. Methods to produce rapid quantitative images include the elegant but computationally complex Hilbert microscopy technique [3], which involves reconstructing the analytic signal from an interferogram Parallel phase stepping, where four phase steps are displayed simultaneously, can also be effective, although at a considerable increase in the complexity of both the optics and the processing algorithms [4].

Wide-field heterodyne interferometry has been previously performed within our group [5], and while this produced quantitative images, the image acquisition speed was limited to one quarter the frame rate of the CCD camera, which was too slow to overcome the deleterious effects of microphonic vibrations. In this Letter, we show how the problems encountered with previous techniques can be overcome with the use of a new custom camera and an appropriate reconstruction procedure.

Phase reconstruction algorithm: A conventional two-beam interference pattern can be written as

$$
i=b^{2}+k \cos \left(\phi_{\mathrm{s}}-\phi_{\mathrm{r}}\right)
$$

where $b^{2}=\left|E_{\mathrm{s}}\right|^{2}+\left|E_{\mathrm{r}}\right|^{2}$ and $k=2\left|E_{\mathrm{s}}\right|\left|E_{\mathrm{r}}\right|$; subscripts $\mathrm{r}$ and s represent the reference and signal beams; $E$ is the field strength of the electromagnetic wave and $\phi$ denotes the phase. In many cases, including biological and engineering samples, the phase distribution $\phi_{\mathrm{s}}$ is the most relevant parameter. For a heterodyne system [5], $\phi_{\mathrm{r}}$ can be replaced by a continuously varying phase shift $\Delta \omega t$, where $\Delta \omega$ is the frequency difference between the reference and sample beams. A more compact setup is to use an electro-optic or a photoelastic modulator (EOM or PEM) to modulate $\phi_{\mathrm{r}}$ sinusoidally. This is referred to as 'pseudo' heterodyne. The phase modulation is given by

$$
\phi_{\mathrm{r}}=p \cos (\Omega t+\alpha)
$$

where $p$ is the phase modulation index (radians); $\Omega=2 \pi f_{\mathrm{m}}$ is the modulation angular frequency and $\alpha$ is the initial phase of the local oscillator relative to the phase of the modulation. Since our detector integrates the signal to extract $\phi_{\mathrm{s}}$, we integrate the signal over four phase intervals (these can take known but arbitrary values, but $\pi / 2$ was used to match the experiments). We generate the numerically derived functions

$$
\begin{aligned}
& A_{n}(p, \alpha)=\int_{(n-1) \pi / 2}^{n \pi / 2} \cos [p \cos (\Omega t+\alpha)] \mathrm{d} t \\
& B_{n}(p, \alpha)=\int_{(n-1) \pi / 2}^{n \pi / 2} \sin [p \cos (\Omega t+\alpha)] \mathrm{d} t
\end{aligned}
$$

These are related to Bessel functions but the $A$ and $B$ functions can be evaluated for arbitrary intervals for any value of $\alpha$ and can furthermore be used to compensate for any imperfections in the modulation scheme. After a simple normalisation to the mean value, the integrated output from the interferometer, $i_{n}$, can be written as

$$
i_{n}=1+k^{\prime} A_{n}(p, \alpha) \cos \phi_{\mathrm{s}}+k^{\prime} B_{n}(p, \alpha) \sin \phi_{\mathrm{s}}
$$

We can reconstruct the sample image to obtain $k^{\prime}$ and $\phi_{\mathrm{s}}$ using the measured values of $i_{n}$ using the approach given in [6]

$$
\left[\begin{array}{ccc}
4 & \sum_{n} A_{n} & \sum_{n} B_{n} \\
\sum_{n} A_{n} & \sum_{n} A_{n}^{2} & \sum_{n} A_{n} B_{n} \\
\sum_{n} B_{n} & \sum_{n} A_{n} B_{n} & \sum_{n} B_{n}^{2}
\end{array}\right]\left[\begin{array}{c}
1 \\
r \cos \phi_{\mathrm{s}} \\
r \sin \phi_{\mathrm{s}}
\end{array}\right]=\left[\begin{array}{c}
\sum_{n} i_{n} \\
\sum_{n} A_{n} i_{n} \\
\sum_{n} B_{n} i_{n}
\end{array}\right]
$$

The modulation index $p$ and the initial phase $\alpha$ affect the quality of reconstructed images. When $\alpha$ is close to 0 , the $3 \times 3$ matrix in (6) is badly conditioned. We have determined numerically that the best condition number can be achieved when $p \approx 2.4482$ at $\alpha= \pm 45^{\circ}$, but if $p$ is kept within $10 \%$ of this value and $\alpha$ is maintained between $40^{\circ}$ and $50^{\circ}$ then the RMS phase noise is never more than $50 \%$ worse than the RMS noise for true heterodyne interferometry with similar a input signal-to-noise ratio. This modulation index was easily achieved in our experiment.

Fig. 1 shows a schematic of the wide-field pseudo-heterodyne imaging system. The light source was a single-mode fibre-coupled super luminescent diode of wavelength $680 \mathrm{~nm}$. It is collimated by a 0.55 numerical aperture (NA) lens L1 and then passes through polariser $\mathrm{P} 1$ which is aligned at $45^{\circ}$ with respect to the axis of the EOM. The EOM acts as an electronically tunable waveplate whose modulation amplitude depends on the control signal voltage. An achromatic lens L2 focuses the beam onto the back focal plane of two objectives with $\times 20,0.4$ NA (sample arm) and $\times 100.2$ NA (reference arm) performing uniform illumination at the sample and reference planes. Interference between the sample and reference beams occurs at the detector after passing through the second polariser $\mathrm{P} 2$, also oriented at $45^{\circ}$ with respect to the EOM.

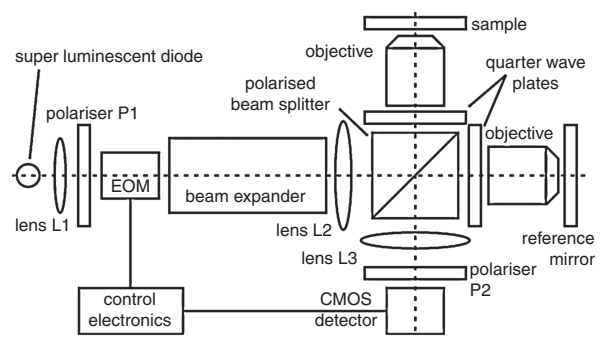

Fig. 1 Schematic of wide-field interferometer

Detector: The key element of the system is the complementary metaloxide semiconductor (CMOS) phase-sensitive detector array. Fig. 2 shows a schematic of a single pixel element and the associated timing diagram, which consists of a photodiode and four storage capacitors, each of which has an independent shutter control. The device is a modified version of the well-known active pixel sensor architecture commonly used in CMOS sensors. The camera was made using a $0.35 \mu \mathrm{m}$ 2P4M CMOS process, has $64 \times 64$ pixels with four storage capacitors at each pixel and a pixel pitch of $30 \mu \mathrm{m}$. Pixels can be randomly addressed, with all four analogue outputs buffered and multiplexed at the column level with a simple differential pair buffer, followed by a two-stage opamp buffer before the chip output. The chip allows a frame rate of 2000 frames per second and each capacitor can store $\sim 10^{6}$ electrons, considerably more than that provided by the photodiode capacitance alone. A microphotograph of the chip is shown in Fig. 3. The additional capacitors greatly increase the well depth, thus substantially reducing the effects of shot noise. The circuit stores charge on each of the four capacitors for an arbitrary period so that the integration shown in (3) and (4) can be effected. Resetting the diode allows storage at another capacitor for the next integration period. The four capacitors can each be used sequentially to store over an integration period before the camera needs to be read out. This allows for very rapid acquisition of all four phase steps at modulation frequencies of up to $1 \mathrm{MHz}$ as the usual readout bottleneck is avoided. This is in contrast to previous works, where the modulation frequency, and hence the ability to suppress vibration, has been limited to one-third or one quarter of the frame rate. In the results reported here, each capacitor was used to store charge over $1 / 4$ of a modulation cycle. Changing the timing of the shutter signal allows parameter $\alpha$ to be varied. It can be seen that the reconstruction algorithm is well matched to the circuit architecture. 


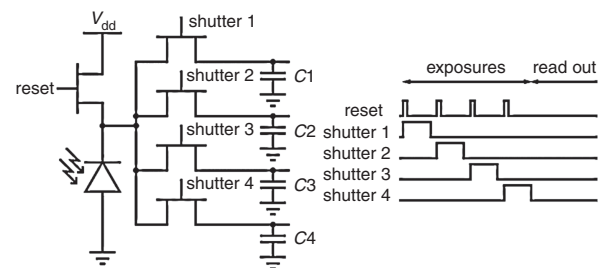

Fig. 2 Schematic of pixel consisting of photodetector and storage capacitors with independent shutter control and timing diagram

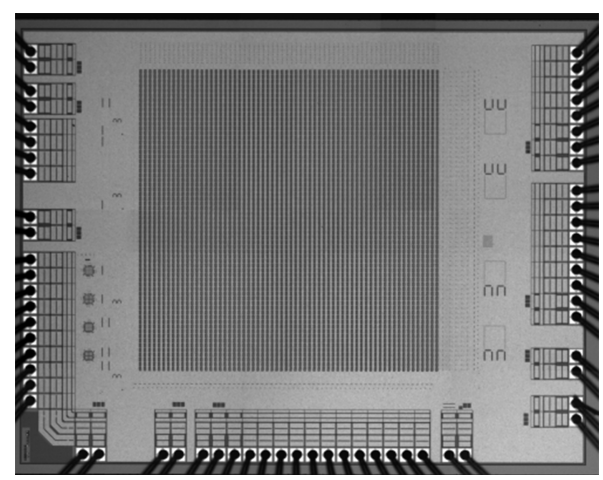

Fig. 3 Microphotograph of CMOS detector (image width $3.8 \mathrm{~mm}$ )

Results: A chromium-coated glass grating of $100 \mathrm{~nm}$ depth and $82 \mu \mathrm{m}$ period was used to test the system and the reconstruction. Using the system described in Fig. 1 and applying the pseudo-heterodyne algorithm, we acquired the reconstructed phase image shown in Fig. 4. The modulation frequency and the modulation index of the EOM were $2 \mathrm{kHz}$ and 2.448 radians, respectively. The phase image shown here was reconstructed with a single frame only.

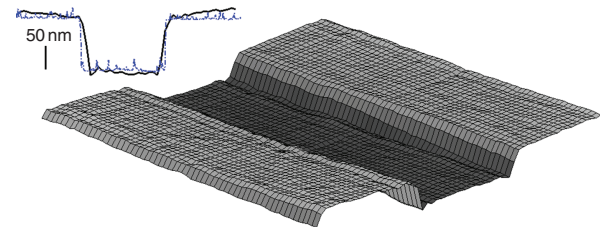

Fig. 4 Reconstructed wide-field phase image and line scan

Solid line scan is from interferometer; dashed line is from profilometer

The ability to acquire all four phase steps very rapidly, without reading the camera, ensured that the effective microphonics within each frame were frozen. For single-shot acquisition, the phase was compared with the weighted average of a reference area $(3 \times 3$ pixels $)$. The weighted average was calculated by averaging the in-phase and quadrature components and converting to a phase value. Although our system freezes noise within a single frame, microphonic effects are still present between frames, so in order to average over several frames to reduce noise it is necessary to provide a common reference for each frame. The phase of the reference area was set to a predefined value and the image pixels were presented relative to the phase of this region, which made averaging over several frames possible and freezes the microphonics for the averaged image. The crucial advantage of the camera is that it allows frames to be acquired relatively free of microphonics and the simple referencing allows averaging between frames. Moreover, the effect of shot noise in each frame is reduced by the large well depth.
To test the camera stability and pixel homogeneity, a series of frames was acquired. The average offset and standard deviation calculated from 45 frames was $0.0051 \pm 0.0012$ radians, which indicates that the noise was $<0.3 \mathrm{~nm}$ in a single-shot acquisition.

Conclusion: We have presented a quantitative, pseudo-heterodyne interference contrast microscope that uses a custom CMOS camera capable of rapidly extracting phase over a full frame. The camera operates sufficiently quickly that the effects of microphonics can be frozen, allowing both single-shot operation and averaging of frames. This has allowed us to perform quantitative interference contrast microscopy without the need for anti-vibration measures and renders the interference technique far more suitable for general use where anti-vibration is either not possible or not desirable. The camera can also be used very effectively with a speckle interferometer [7], or to acquire and calculate the fundamental and second-harmonic components of a sinusoidally phase modulated signal. This can be useful in experimental techniques that use a PEM or an EOM to modulate the incident light.

Acknowledgment: The authors acknowledge the support of the Engineering and Physical Sciences Research Council (EPSRC), UK.

(C) The Institution of Engineering and Technology 2015

22 January 2015

doi: $10.1049 / \mathrm{el} .2015 .0268$

One or more of the Figures in this Letter are available in colour online.

J. Zhang, C.W. See, R.J. Smith, N.S. Johnston, M.C. Pitter, M.G. Somekh and R.A. Light (Institute of Biophysics, Imaging and Optical Science, University of Nottingham, University Park, Nottingham, NG7 2RD, United Kingdom)

$\bowtie$ E-mail: roger.light@nottingham.ac.uk

N.S. Johnston: Also with the Centre for Electronic Imaging, Department of Physical Sciences, The Open University, Milton Keynes, United Kingdom.

M.C. Pitter: Also with the Zephir Lidar, Hollybush, Ledbury, United Kingdom.

M.G. Somekh: Also with the Department of Electronic \& Information Engineering, The Hong Kong Polytechnic University, Kowloon, Hong Kong.

\section{References}

1 Murphy, D.: 'Phase contrast microscopy', in 'Fundamentals of light microscopy and electronic imaging' (Wiley-Liss, New York, 2001)

2 Gundlach, H.: 'Phase contrast and DIC instrumentation and applications in cell, developmental, and marine biology', Proc. SPIE, 1994, 1846, pp. 126-139

3 Ikeda, T., Popescu, G., Dasari, R.R., and Feld, M.S.: 'Hilbert phase microscopy for investigating fast dynamics in transparent systems', Opt. Lett., 2005, 30, (10), pp. 1165-1167

4 Sawyer, N.B.E., Morgan, S.P., Somekh, M.G., See, C.W., Cao, X.F., Shekunov, B.Y., and Astrakharchik, E.: 'Wide field amplitude and phase confocal microscope with parallel phase stepping', Rev. Sci. Instrum., 2001, 72, (10), pp. 3793-3801, doi: 10.1063/1.1400156

5 Pitter, M.C., See, C.W., and Somekh, M.G.: 'Full-field heterodyne interference microscope with spatially incoherent illumination', Opt. Lett., 2004, 29, (11), pp. 1200-1202, doi: 10.1364/OL.29.001200

6 Grievenkamp, J.E.: 'Generalized data reduction for heterodyne interferometry', Opt. Eng., 1984, 23, pp. 350-352

7 Somekh, M.G., See, C.W., and Goh, J.: 'Wide field amplitude and phase confocal microscope with speckle illumination', Opt. Commun., 2000, 174, (1-4), pp. 75-80, doi: 10.1016/S0030-4018(99)00657-4 